\title{
PIV-based dynamic model of EHD volume force produced by a surface dielectric barrier discharge
}

\author{
N. Benard ${ }^{\ddagger 1}$, S. Laizet ${ }^{\S, * 2}$, and E. Moreau ${ }^{\ddagger 3}$ \\ ${ }^{\ddagger}$ Institut PPRIME - UPR 3346 - CNRS - Université de Poitiers - ISAE/ENSMA - SP2MI Téléport2 Bd Marie \& Pierre Curie \\ BP 30179, 86962 Futuroscope Chasseneuil Cedex, France \\ ${ }^{\S}$ Imperial College of London, London, SW7 2AZ, UK
}

In this paper, an experimental measurement of the flow produced by a surface DBD plasma actuator has been conducted. One original aspect of these measurements by particle image velocimetry is the high acquisition rate for a PIV system $(20 \mathrm{kHz})$. By using these highlyresolved flow measurements, the fluid flow velocity is used to estimate the spatial and temporal evolution of the EHD volume force. A reduced order model of this force has been constructed by proper orthogonal decomposition. Based on the analysis of the time-resolved expansion coefficients and their associated spatial modes, it is shown that the volume force can be reconstructed by using a limited number of POD modes (6 modes). This spatial and temporal filtering of the force fields remains faithful to the original data and it will help in view of an implementation of such a source term in a numerical solver. The resulting dynamic model shows an alternation of positive and negative volume forces. The strong positive EHD force developing in the glow regime of the DBD plasma discharge is visualized in a time-resolved manner. This positive force is immediately followed by a strong negative volume force probably caused by the local flow deceleration.

\section{Introduction}

$\mathrm{T}^{\mathrm{s}}$ The magnitude, spatial distribution, and time evolution of the body force produced by plasma discharge propagating over dielectric wall are essential mechanical characteristics when these discharges are used to superimpose periodic perturbations to a fluid flow in view of its manipulation. A deep knowledge of these quantities can help in optimizing this type of electrical discharge by experiments and it can provide the information for defining a relevant model of plasma actuator. Here, the proposed study relates to the well-known dielectric barrier discharge plasma actuator where two conductive electrodes are placed asymmetrically on both sides of a dielectric barrier [1-4]. In a basic view, because of the alternative high amplitude electric field, gas ionization occurs and the displacement of the charged particles under the influence of the electric field transfers fluid momentum through a volume force referred as electrohydrodynamic (EHD) force. In fact, such an electrical discharge involves electrical, mechanical, thermal and chemical mechanisms and is a complex multi-physics problem [5]. Numerical modelling of these discharges is not an easy task. In order to model the physical mechanisms of a plasma actuator first principle based methods can be used. By computing the solution of complex transport equations for both charged and neutral species, the solution of a Poisson equation for the electric field, and finally coupling the EHD force to the fluid by using Navier-Stokes equations, the electromechanical conversion of plasma actuators can be modeled [6-8]. This approach is complex and time-consuming for non-parallelized code, limiting its

\footnotetext{
${ }^{1}$ Nicolas Benard, Associate Professor, nicolas.benard@univ-poitiers.fr, AIAA senior member

${ }^{2}$ Sylvain Laizet, Lecturer, Department of Aeronautics, Imperial College London, UK

${ }^{3}$ Eric Moreau, Professor, University of Poitiers
} 
use to a few ac cycles only (and preferentially high ac frequency) and to strictly 2D discharge and flow. However, due to the development of parallel and distributed hardware architectures, the inhomogeneous space charge distribution characterizing 3D discharge can now be numerically modeled [9]. Another approach consists of implementing a simplified model of the body force produced by the discharge as a source term in a CFD solver [10]. Commonly used models are based on a body force distribution from the solution of a Laplace's equation for electric potential due to the voltage applied to the electrodes and a Poisson like second order partial differential equation for the charge density of the ionized working fluid [11-12]. This type of model has to assign a certain charge distribution at the dielectric wall as a boundary condition in the solver. Steady Gaussian distribution is sometime used as in [11] but the phenomenon of charge deposition in DBD is unsteady and varies in space by nature [13]. The charge deposition contributes largely to the physics of the plasma discharge by its screening effect and its involvement in the mutual influence between half-cycle to half-cycle plasma regimes. However, the simplified models can be modified and a time dependent solution of the charge density can be implemented as in [14] or [15].

The magnitude of the unsteady EHD body force can be experimentally evaluated from fluid momentum equations in integral form [16-17], but the spatial distribution of the body force can also be experimentally determined using advanced optical techniques such as particle image velocimetry (PIV) measurements (indirect method [18]). Such an approach can provide relevant PIV-based model of the EHD volume force, model that can be implemented into solvers resolving the Navier-Stokes momentum equations [19-20]. The most straightforward strategy for evaluating the EHD volume force is based on the two-dimensional incompressible Navier-Stokes equations. Assuming that the pressure gradients are much smaller that the force components, they can be neglected as initially proposed in [21] and because all the other components of the Navier-Stokes equations can be computed from the experimental data, the 2D spatial distribution of the mean force component can be evaluated fairly easily [22- 25]. In most of the cases, time-averaged velocity fields are measured, but phase-averaging procedure can also be used to recover the dynamic aspects of the EHD volume force [26-27]. Another method consists of measuring the produced flow by time-resolved PIV and by computing the corresponding time-resolved volume force as recently reported in [28]. This type of time-resolved evaluation of the volume force distribution can be helpful to define a reduced order model of the EHD force production. By using typical methods such as a proper orthogonal definition, a simplified dynamic model should be defined.

The main objective of the present study is to define a dynamic model of the EHD volume force produced by a surface plasma discharge. By using particle image velocimetry at high acquisition frequency $(20 \mathrm{kHz})$, the distribution in space and time of the EHD volume force is extracted from the Navier-Stokes equations. Different methods are presented to improve the quality of the volume force fields. In particular, the study focuses on the construction of a reduced order model based on proper orthogonal decomposition applied to time-resolved $2 \mathrm{D}$ vector fields.

\section{Experimental setup}

\section{II-1 DBD actuator configuration}

PIV measurements have been conducted for different voltages (from 12 up to $20 \mathrm{kVamp}$ ) and frequencies (from $100 \mathrm{~Hz}$ to $2000 \mathrm{H} \mathrm{z}$ ) in order to characterize the effect of a DBD plasma actuator in an ambient fluid from initially at rest condition. The DBD actuator consists of two aluminum electrodes asymmetrically mounted on a 3-mm thick dielectric plate made of PMMA. All its dimensions are indicated in figure 1. A signal amplifier (Trek, model $30 / 40 \mathrm{~mA}$ ) is used to apply a gain of $3,000 \mathrm{~V} / \mathrm{V}$ to the input voltage provided by a function generator. The input signal is a sine 
waveform applied to the top-electrode while the bottom electrode is grounded and encapsulated. In this paper, all the proposed results concern a sine waveform with amplitude of $20 \mathrm{kV}$ and ac frequency $\mathrm{f}_{\mathrm{AC}}$ equal to $1000 \mathrm{~Hz}$.

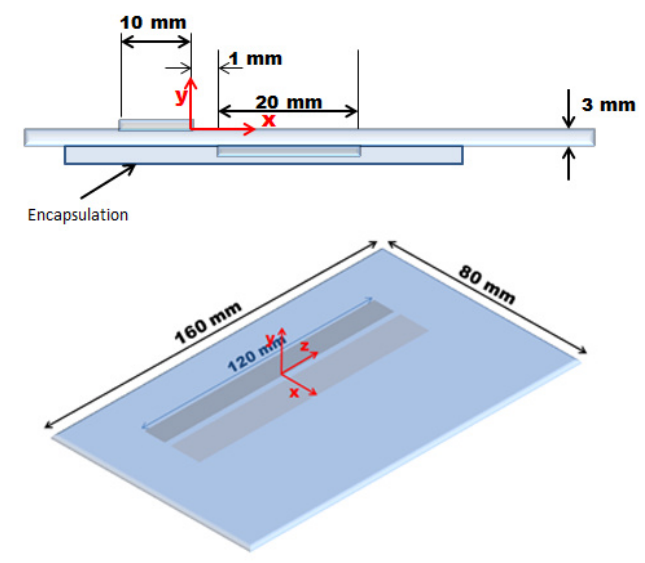

Figure 1. Schematic illustration of the surface DBD plasma actuator.

\section{II-2 Description of the fluid flow measurements}

The time-resolved velocity produced at the center of the actuator $(\mathrm{z}=0)$ is measured by a PIV system triggered by the applied voltage signal. The PIV system is composed of dual-head Nd-YAG laser (Terra-PIV, Continuum), a fast CCD camera with $1024 \times 1024$ resolution (Fastcam SA-Z, Photron), a trigger unit and a multi-core PC running Davis 8.2 PIV software. The flow is seeded with droplets of dielectric oil (Ondina 919) with a particle diameter equal to $0.3 \mu \mathrm{m}$. The velocity fields are obtained by a cross correlation algorithm with adaptive multi-pass $(32 \times 32$ to $16 \times 16$ with $50 \%$ overlapping interrogation windows). The camera and laser are operated at $20 \mathrm{kHz}$ and series of 88000 images are recorded for each test. Because of the synchronization between the electrical signal and the fluid response, the precise arrival time of all images is known and a phase-averaging procedure can be applied in order to reconstruct the flow dynamics.

\section{Volume force estimation}

The direct information obtained from the PIV is the instantaneous vector fields that can be averaged in time. The flow caused by the surface DBD actuator is depicted in Figure 2 by using separated plot for the $\mathrm{u}$ and $\mathrm{v}$ velocity magnitudes. The vector fields indicate a typical wall jet that forms from the topelectrode and directed toward the grounded one. The maximum velocity amplitude reaches $6 \mathrm{~m} / \mathrm{s}$ close to the end of the plasma extension (here at $\mathrm{x} \sim 10 \mathrm{~mm}$ by considering the applied voltage and frequency) and a quick jet spreading is observed in the u velocity component. The transverse flow velocity component has a smaller amplitude that the $u$ velocity component and two main regions are reported. The first one corresponds to the suction of the flow in quiescent condition above the topelectrode, this suction producing a strong velocity with a magnitude of about $-0.9 \mathrm{~m} / \mathrm{s}$. The second region relates to a fluid ejection above the plasma discharge (magnitude of about $0.4 \mathrm{~m} / \mathrm{s}$ ). The Figure 3 also includes the spatial distribution of the velocity fluctuations u'u', v'v' and u'v' averaged in time (Reynolds stress components). As for the transverse velocity component, two regions are highlighted. The first one concerns the suction above the top-electrode where strong velocity fluctuations are responsible for the high amplitude of the Reynolds stress tensor. The second region corresponds to the shear layer caused by the interface between the region of high speed and the surrounding flow in quiescent condition. The larger fluctuations are in the streamwise direction due to the periodic pushing effect caused by the EHD body force. 

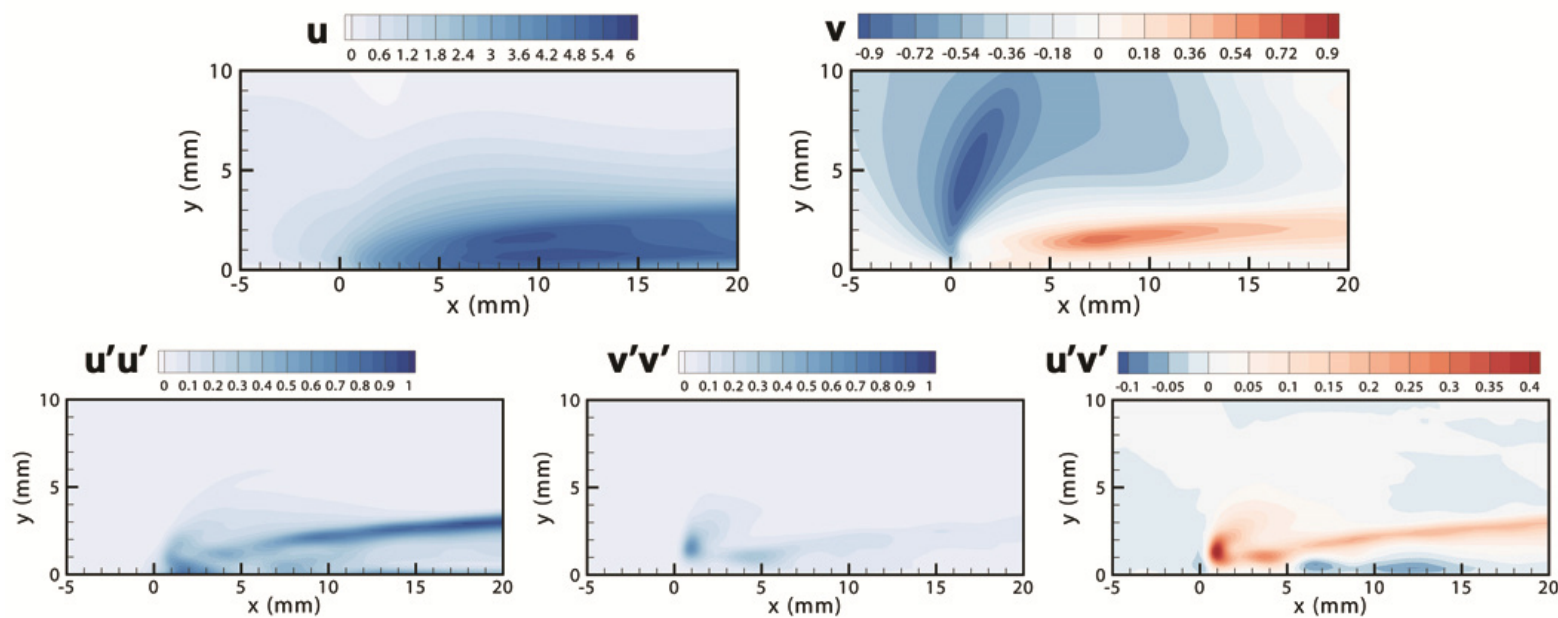

Figure 2. Time-averaged velocity flow produced by the surface DBD operated at $20 \mathrm{kV}$ and $1000 \mathrm{~Hz}$.

The PIV system operates at high-speed $(20 \mathrm{kHz})$, this meaning that a time-resolved description of the flow field can be obtained. The local flow velocity at two measurement points is plotted in Figure 3 , one location being close to the top-electrode where the EHD force should be high $(x=1 \mathrm{~mm}$ and $\mathrm{y} \sim 100 \mu \mathrm{m})$ and the second one just above the plasma discharge $(\mathrm{x}=6 \mathrm{~mm}, \mathrm{y}=1.5 \mathrm{~mm})$. Both plots show a periodic flow with fluctuations at the imposed $\mathrm{f}_{\mathrm{AC}}$ frequency. The amplitude of the peak velocity is higher further downstream of the top-electrode because the momentum transfer needs some space to be fully developed but a higher periodicity is observed close to the electrode acting as ionizer. The behavior of the flow velocity produced by the discharge is in agreement with the literature, the velocity being mainly produced during one half of the ac period [2]. This plot clearly indicates that the amplitude of the instantaneous velocity field is not strictly reproducible between two successive periods, this supporting for a dedicated processing of the raw data before defining an EHD volume force model.
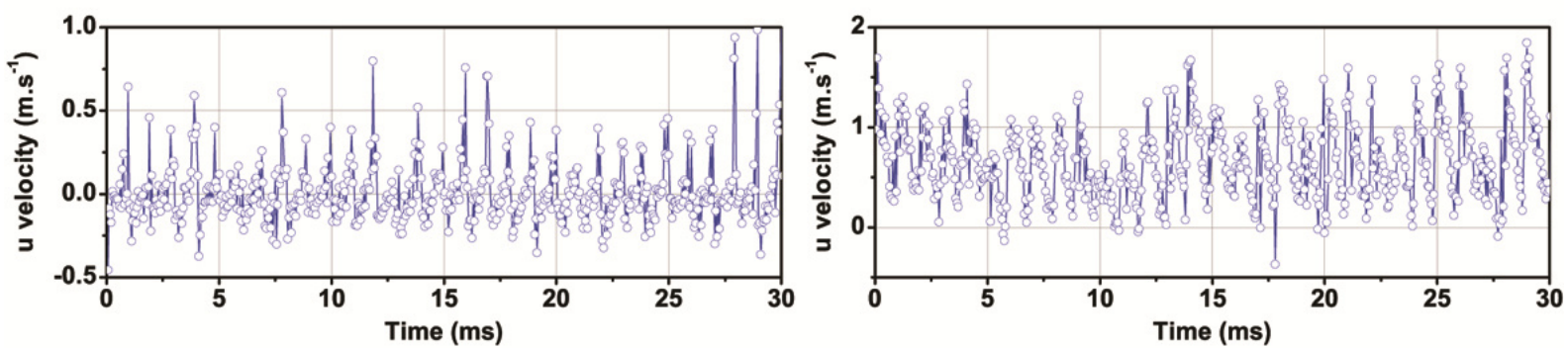

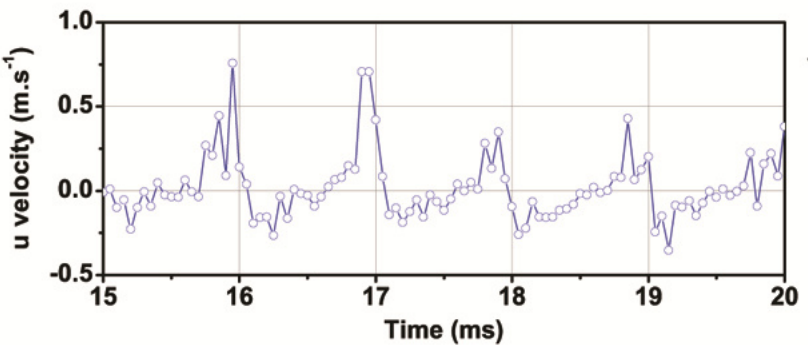

(a)

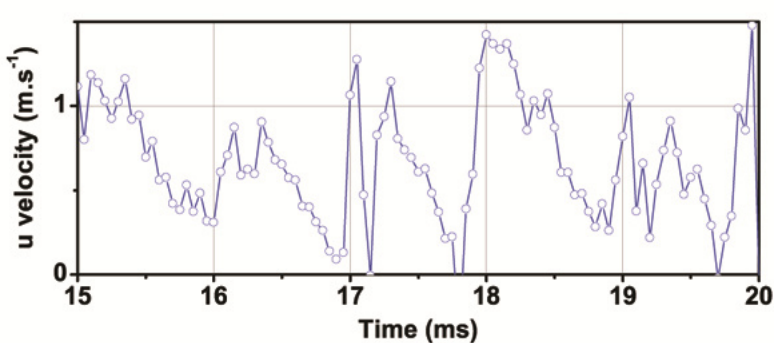

(b)

Figure 3. Time-resolved velocity flow (u component) produced by the surface DBD operated at $20 \mathrm{kV}$ and 1000

Hz. The left plot corresponds to position $\mathrm{x}=1 \mathrm{~mm}$ and $\mathrm{y} \sim 100 \mu \mathrm{m}$ when the right plot refers to velocity measurements at $\mathrm{x}=6 \mathrm{~mm}$ and $\mathrm{y}=1.5 \mathrm{~mm}$. 


\section{III-1 Principle of PIV-based estimation of the volume EHD force}

The mean flow produced by the discharge obeys the incompressible two-dimensional Navier-Stokes equations. At each data point of the measured velocity field, the mean volume force $\mathrm{F}$ can be expressed as

$\mathbf{F}-\nabla p=\rho \mathbf{U} \cdot \nabla \mathbf{U}-v \nabla^{2} \mathbf{U}$

where $\mathrm{p}$ is the pressure field, $\mathrm{U}$ is the mean velocity field while $\rho$ and $v$ are the gas density and gas dynamic viscosity, respectively. The right hand side of this equation can be evaluated at each measurement location from the velocity measurements. It is considered that the pressure contribution is minor regarding the volume force produced by the discharge as in [20, 21, 25, 27]. This point has been discussed in Brauner et al. [28] and it was shown that the pressure gradient can contribute to the mean EHD volume force. The contribution of the pressure gradient in the computation of the EHD force has also been partially discussed in the pioneering work of [22]. It was shown that convection and pressure gradient are initially negligible, but the influence of the pressure gradient can increase with time. This has also been discussed in case of the time-dependent modelling of the EHD volume force from PIV data [25], the pressure gradient being suspected to be responsible for a negative value of the right hand term of equation (1) at some instants of the ac period. For the moment, the pressure gradients cannot be solved and no method for discriminating the contribution of the pressure gradient has been proposed.

Here, the vector fields are time-resolved, this meaning that the Navier-Stokes equation has to consider for the time dependence. Equation (1) can be rewritten as:

$\mathbf{F}-\nabla \mathrm{p}=\rho\left(\frac{\partial \boldsymbol{U}}{\boldsymbol{\partial} \boldsymbol{t}}+\mathbf{U} \cdot \nabla \mathbf{U}\right)-v \nabla^{2} \mathbf{U}$

In this case, the time-resolved vector fields can be used for evaluating the volume force $\mathbf{F}$ with a temporal resolution of $50 \mu \mathrm{s}$. After the evaluation of $\mathbf{F}$ at each time step, its mean value can be easily computed by a time integration over the recorded sequence. This approach has been used to determine the mean $\mathbf{F}$ as depicted in Figure 4. The spatial distribution and amplitude of $\mathbf{F}$ correspond both to the results of the literature $[22,23,24,28]$. For the horizontal force component $F_{x}$, its mean value is strictly positive in the region of the discharge. This component can reach an amplitude up to 3700 $\mathrm{N} . \mathrm{m}^{-3}$, one $\mathrm{mm}$ downstream of the edge of the top-electrode where the electric field is maximal (end of the inter-electrode gap distance and beginning of the grounded electrode). The region of positive volume force $F_{x}$ matches with the region where plasma develops, between $x=1$ and $x=10 \mathrm{~mm}$. A small negative contribution can be observed at the wall surface downstream the region of propagation of the plasma discharge. This region is caused by viscous effects on the dielectric wall downstream of the end of the plasma extension. The spatial distribution of the volume force $\mathrm{F}_{\mathrm{y}}$ is also estimated by using Eq. (2). This component is often considered as negligible in DBD modeling (see [11,23]) but the evaluation conducted here shows that the amplitude of $F_{y}$ can be of a same order of magnitude than $F_{x}$. However, it is correct that the region of significant $F_{y}$ is smaller than the region with high $F_{x}$. The $\mathrm{F}_{\mathrm{y}}$ component is mostly positive, especially at $1 \mathrm{~mm}$ from the top-electrode and it corroborates with the periodic flow ejection from the top-electrode toward the grounded one. A region of negative $\mathrm{F}_{\mathrm{y}}$ is also observed precisely above the top-electrode $(\mathrm{x}=0 \mathrm{~mm})$, this negative $\mathrm{F}_{\mathrm{y}}$ being responsible for the flow suction induced by the plasma discharge. The magnitude of this vertical force can be high, but only in very localized regions and this component presents positive and negative values. As a result, when integrated in space as in [17], the contribution of $F_{y}$ appears to be weak compared to $F_{x}$ but it contributes to the periodic flow fluctuations imparted by this DBD plasma actuator [30]. 
Some successive snapshots of the time-dependent volume force are illustrated in Figure 5. At this time, the main conclusion is that the instantaneous volume force looks really noisy. Indeed, the PIVbased model requires an evaluation of the first and second order derivatives by using finite difference schemes (first order in space and time). Here, time-resolved flow measurements are used and there is no phase averaging procedure compared to [26]. The unsteady behavior of $F_{x}$ measured at $x=1 \mathrm{~mm}$ and $\mathrm{y} \sim 100 \mu \mathrm{m}$ is given in Figure 6 for different time windows. Due to unsteady nature of DBD actuators, a periodic pattern is expected for the EHD volume force. Here, a pattern cannot be easily observed. The present results indicate that the spatial distributions of both components of $\mathbf{F}$ are not smooth enough for an easy implementation in a numerical CFD solver. Indeed, the strong spatial and temporal gradients observed in the time evolution of $\mathrm{F}$ may promote a low convergence rate and may destabilize the numerical schemes used in numerical CFD solvers. This is a consequence of the 'low' quality of the vector fields (caused by a low signal to noise ratio when time-resolved system is used). Finally, it becomes clear that a specific filtering is required to get an accurate description of the volume force distribution and a model suitable for direct numerical simulations or for Unsteady Reynolds-averaged Navier-Stokes solvers.
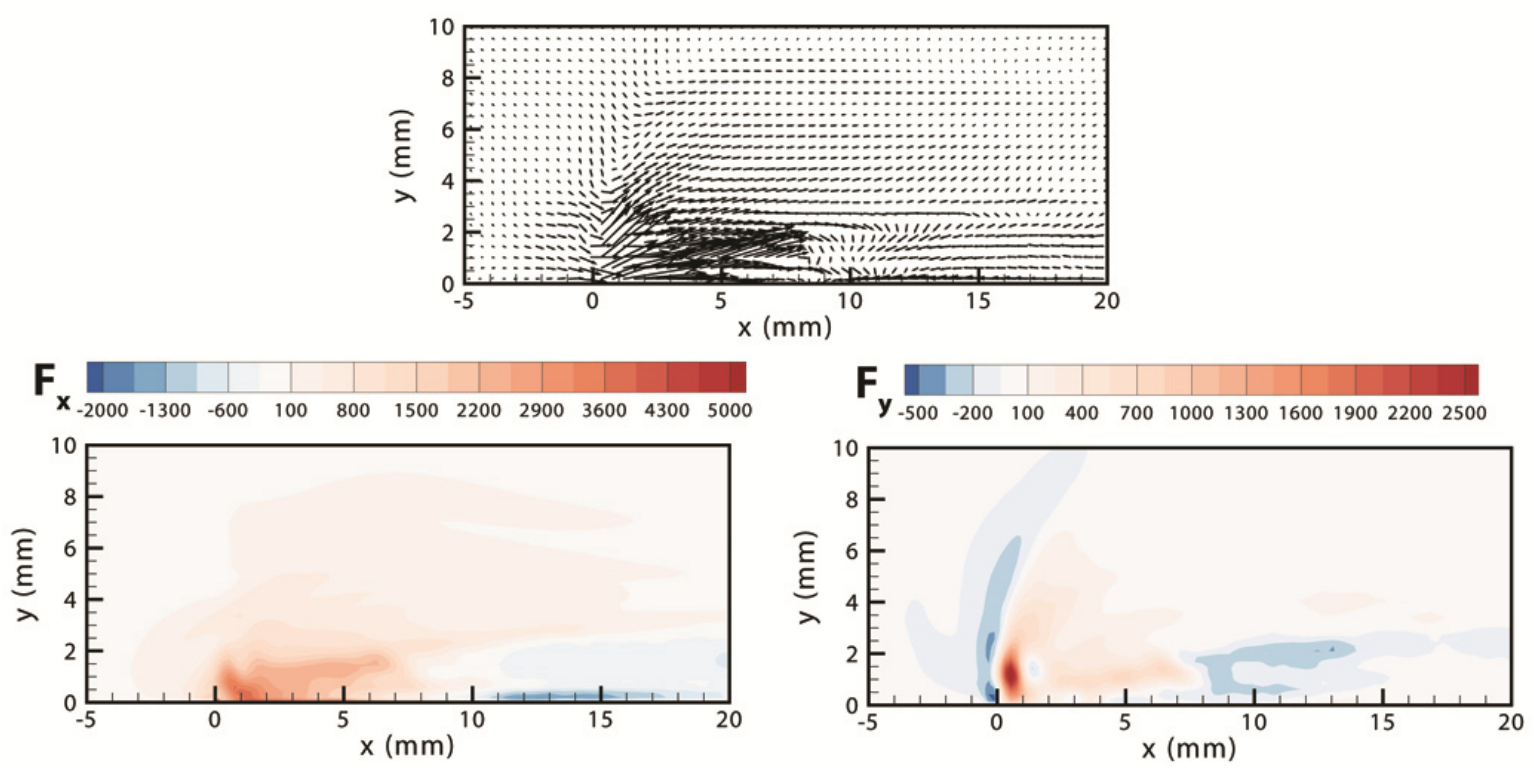

Figure 4. Spatial distribution of the mean volume force as vector field, $F_{x}$ force component and $F_{y}$ component. Volume force in $\mathrm{N} / \mathrm{m}^{3}$ for an applied voltage amplitude of $20 \mathrm{kV}$ and $\mathrm{f}_{\mathrm{AC}}=1000 \mathrm{~Hz}$. 

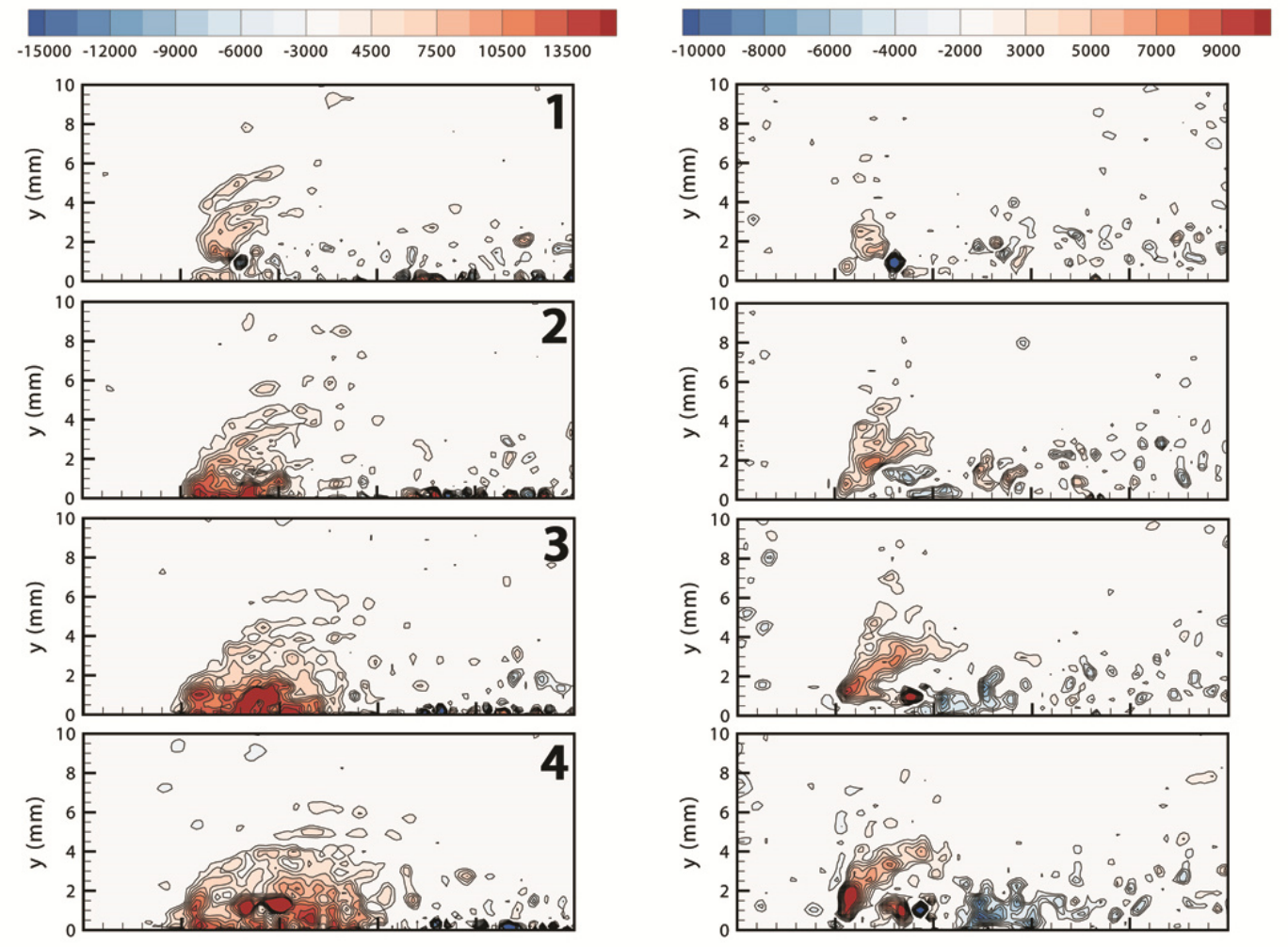

Figure 5. Spatial distribution of instantaneous volume force at 4 successive instants separated by $50 \mu$ s. Force in $\mathrm{N} / \mathrm{m}^{3}$ for an applied voltage amplitude of $20 \mathrm{kV}$ and $\mathrm{f}_{\mathrm{AC}}=1000 \mathrm{~Hz}$.
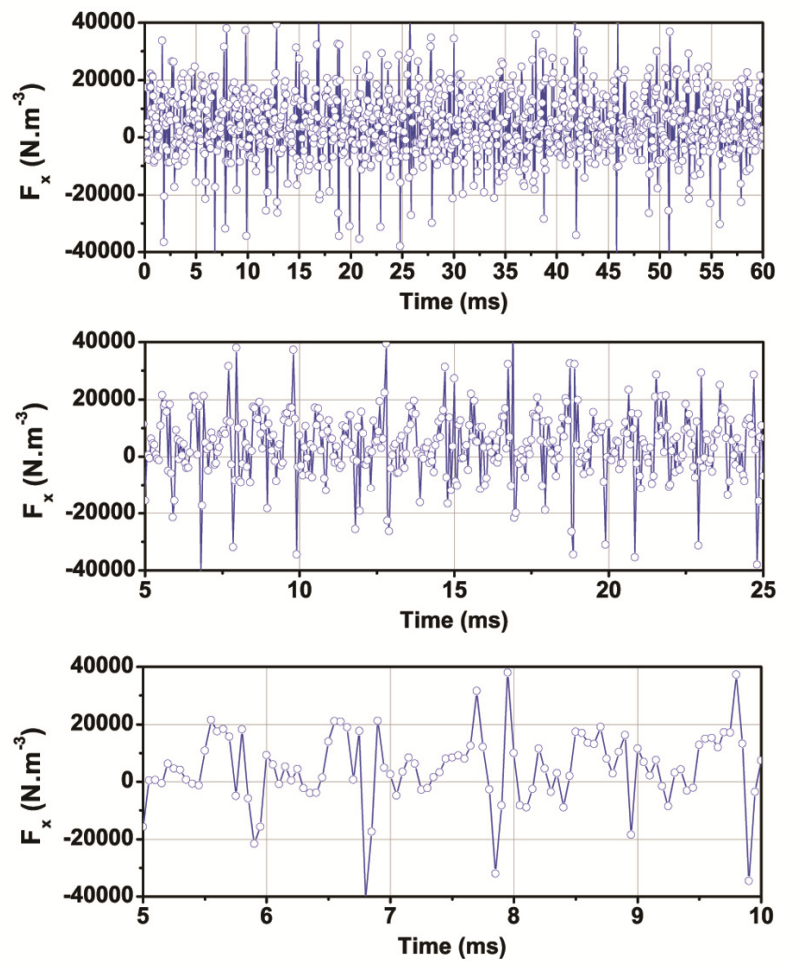

Figure 6. Time-resolved EHD volume force (x component) measured at $\mathrm{x}=1 \mathrm{~mm}$ and $\mathrm{y} \sim 100 \mu \mathrm{m}$. 


\section{Construction of a reduced order model}

As it was shown in the previous section, the volume force estimated by the PIV velocity information is particularly noisy in space and time. Here, a reduced order model of the EHD volume force by proper orthogonal decomposition (POD) is considered in order to extract the dynamic of the plasma actuator while reducing the measurement noises. In practice POD is implemented discretely and based on the fluctuating components of the EHD volume force $\mathbf{F}$ (the mean $\mathbf{F}$ is removed from each instantaneous F). Each instantaneous force field (15000 consecutive snapshots) at a discrete time $t$ is considered in the POD procedure. A full mathematical description of POD can be found in [31-32]. Only a partial view of the method is now described. The instantaneous volume force field (minored by the mean volume force) due to the $\mathrm{n}^{\text {th }}$ POD mode can be written as:

$F_{i}^{n}(X, t)=a_{n}(t) \phi_{i}^{n}(X)$

where $a_{n}(t)$ correspond to the expansion coefficients and $\phi_{i}^{n}$ is the $\mathrm{n}^{\text {th }}$ spatial mode. The spatial POD modes are obtained by numerically resolving an eigenvalue problem:

$$
\sum_{n=1}^{N} R_{i j}\left(t, t^{\prime}\right) a_{n}\left(t^{\prime}\right)=\lambda_{n} a_{n}(t)
$$

where $\mathrm{R}_{\mathrm{ij}}$ is a two-point temporal correlation function and $\lambda_{n}$ are the eigenvalues that represent the energy contained by each POD mode. The kernel defined by the temporal correlation function is numerically solved by a F90 program (64 bits Intel fortran compiler with LAPACK library). The spatial modes $\Phi$ are derived from the expansion coefficients and the local fluctuating velocity by using:

$\phi_{i}^{n}(x, y)=\frac{1}{N \lambda_{n}} \sum_{t=1}^{N} F_{i}^{t}(x, y) a_{n}(t)$

By using POD decomposition, an instantaneous volume force field $\mathrm{F}_{\mathrm{i}}(\mathrm{X}, \mathrm{t})$ can be reconstructed by considering the following expression :

$$
F_{i}(X, t)=\sum_{n=1}^{N} a_{n}(t) \phi_{i}^{n}(X)
$$

The decomposition is used here as a two-dimensional energy-based filtering. Indeed, the useful content of the signal $\mathrm{F}$ can be separated by a truncation of the number of POD modes used to reconstruct the volume force field and finally defined a low order model of the EHD volume force dynamic. The instantaneous volume force is decomposed in:

$$
\begin{aligned}
& \tilde{F}_{i}(X, t)=\sum_{n=1}^{M} a_{n}(t) \phi_{i}^{n}(X) \\
& \text { and } F_{i}^{\prime}(X, t)=\sum_{n=M+1}^{N} a_{n}(t) \phi_{i}^{n}(X)
\end{aligned}
$$

where $\mathrm{M}$ is a suitable cut-off energy level and $\mathrm{N}$ the total number of PIV field considered (here $\mathrm{N}=15000$ ). The primary objective of this investigation is the determination of the expansion coefficients and their associated spatial modes. An analysis of these parameters is provided in order to accurately select a suitable cut-off energy level M.

\section{III-1 Determination of the suitable cut-off energy level}


The contribution of each POD modes on the total energy is plotted in figure 7. The energy distribution spreads over many modes but after the $30^{\text {th }}$ mode, all the modes contribute equally to the total turbulent produced by the estimated EHD force. However, a cut-off level determined only on the energy content of the spatial mode is not sufficient to isolate the relevant signal and minimize the measurement noise.

Because, the original PIV data are resolved in time, the expansion coefficients $a_{n}$ can help in discriminating the most energetic content of the force. The temporal evolution of $a_{n}$ is shown in Figure 8. The temporal signature of the first 5 expansion coefficients presents a good periodicity at $1 \mathrm{kHz}$, this being particularly noticeable in the temporal evolution of the most energetic mode (mode \#1). The good agreement between the applied ac frequency and the response of the volume force can help in determining an accurate truncation for a pertinent volume force reconstruction. Furthermore, in view of the use of the volume force as a source term in numerical solver, its time evolution has to be reduced at one single period. The total sequence of expansion coefficients $(750 \mathrm{~ms}$ here) is phaseaveraged (Figure 9). Indeed, in the present investigation the acquisition rate is constant (20 kHz), and the data can be easily ordered according to a reference time. One period of the plasma discharge covers 1 millisecond $\left(\mathrm{f}_{\mathrm{AC}}=1000 \mathrm{~Hz}\right)$ and, due to the acquisition rate, it can be discretized into 20 instants (i.e. time resolution of $50 \mu \mathrm{s}$ ). The temporal expansion coefficients can be classified according to their arrival time and all the coefficients in a class are then averaged. This results in a temporal filtering of the data. One can calculate the standard deviation for the averaging process in a single class as well as the mean standard deviation for the complete cycle, this leading to a global standard deviation per expansion coefficient. This mean standard deviation is a good indicator of the periodicity of the expansion coefficients (the EHD volume force is expected to be highly periodic), for this reason this quantity is also plotted in Figure 9 for the first $1000 a_{n}$. The sixth first expansion coefficients have a good temporal repeatability as highlighted by the corresponding low mean standard deviation when the $a_{n}$ of these modes are phase-averaged. When they are phase-averaged, the amplitude of these six $a_{n}$ is high compared to the coefficients of higher order, this indicating that their contribution to the unsteady character of the volume force should be considered in a reconstructed model. In order to highlight possible coupling between the POD modes, 2D projection of the phase portrait (plot of the two coefficients series $a_{n}$ and $a_{n+1}$ ) are shown in Figure 10. The plot corresponding to the two first POD coefficients are not independent and they describe a Lissajou curve. This relates to a frequency relation between these two modes, the mode 2 being an harmonic of mode 1 . Due to its high relative energy and excellent periodicity, the first POD mode is the primary contribution to the periodic EHD volume force but it cannot be used alone because of its relationship with mode 2. For the mode of higher order, their portrait phases indicate that they are statistically independent.

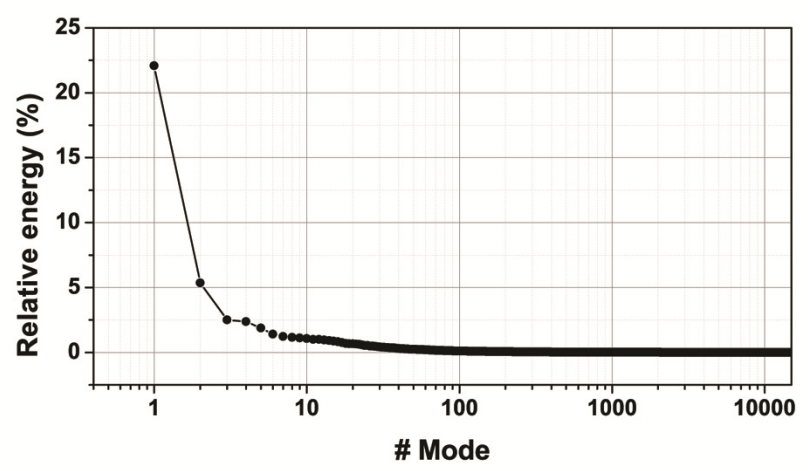

Figure 7. Energy contribution (in percent) of each POD spatial modes 

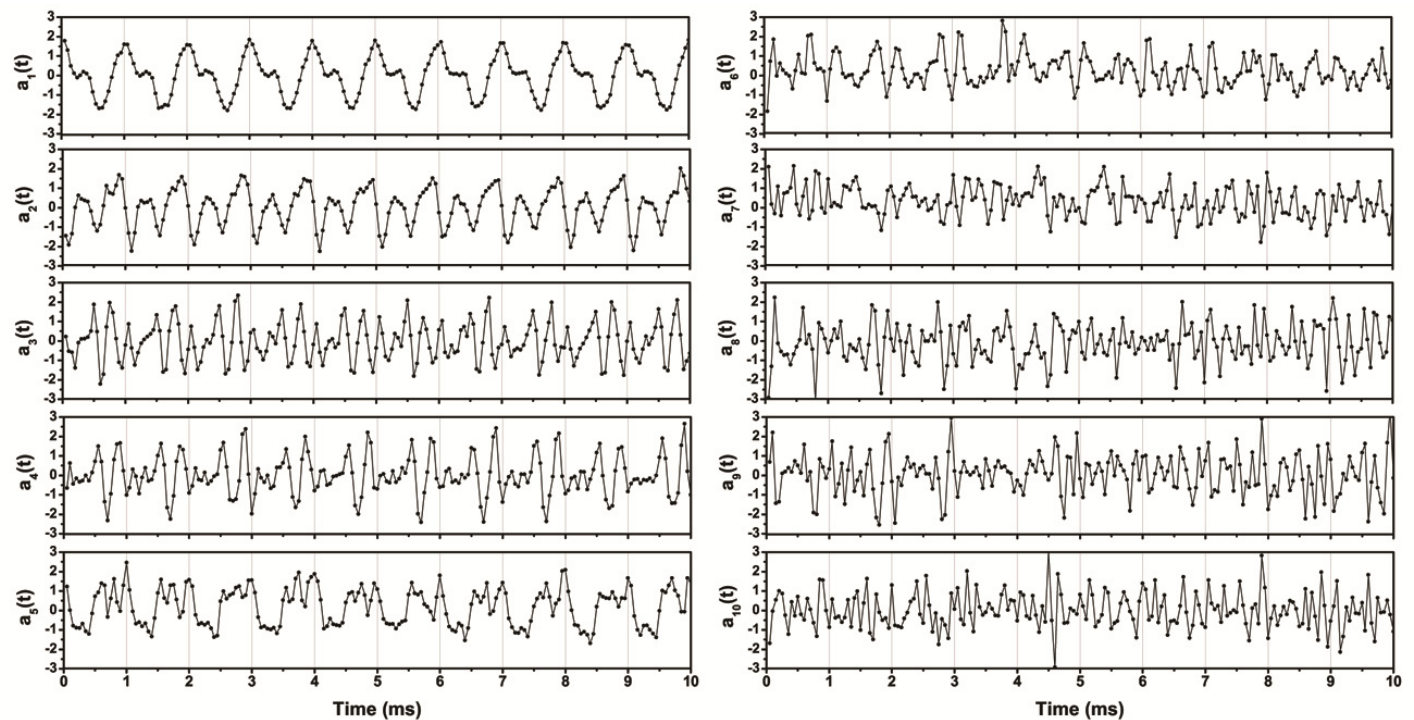

Figure 8. Time evolution of the expansion coefficients $a_{n}$ (with $n$ from 1 to 10 ).
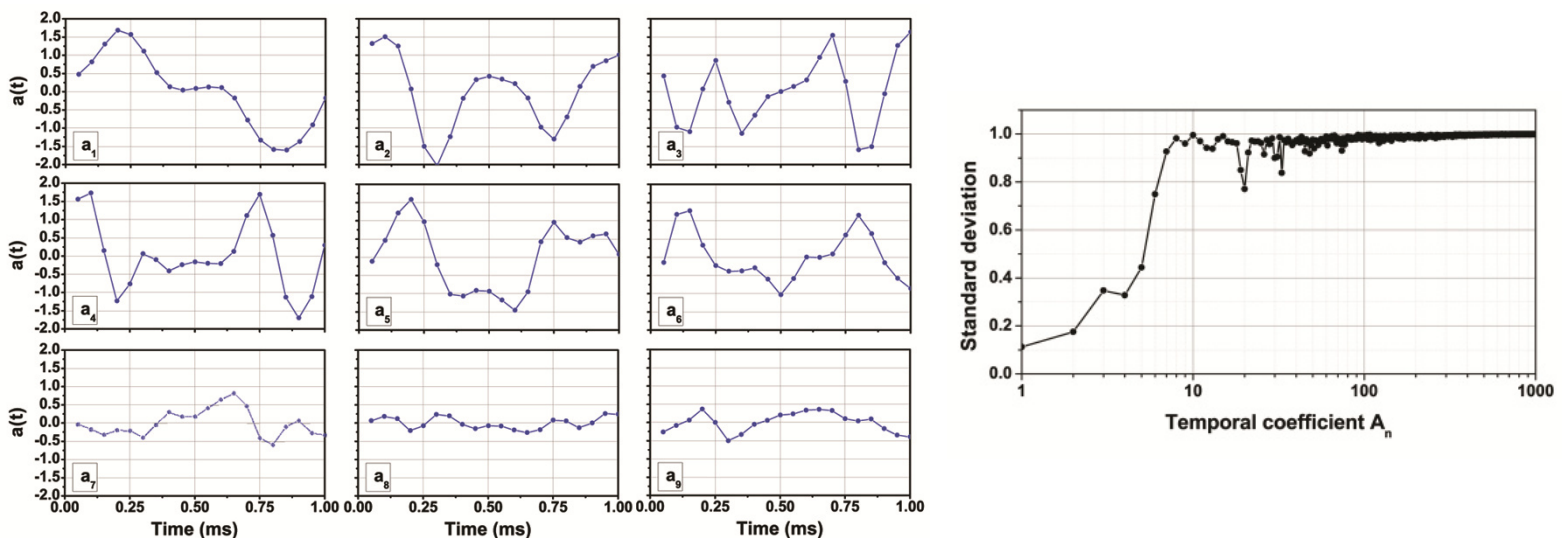

Figure 9. Phase-averaged expansion coefficients and mean standard deviation in the phase-averaging procedure for 1000 expansion coefficients. By construction the sign of the $a_{n}$ has no physical meaning.
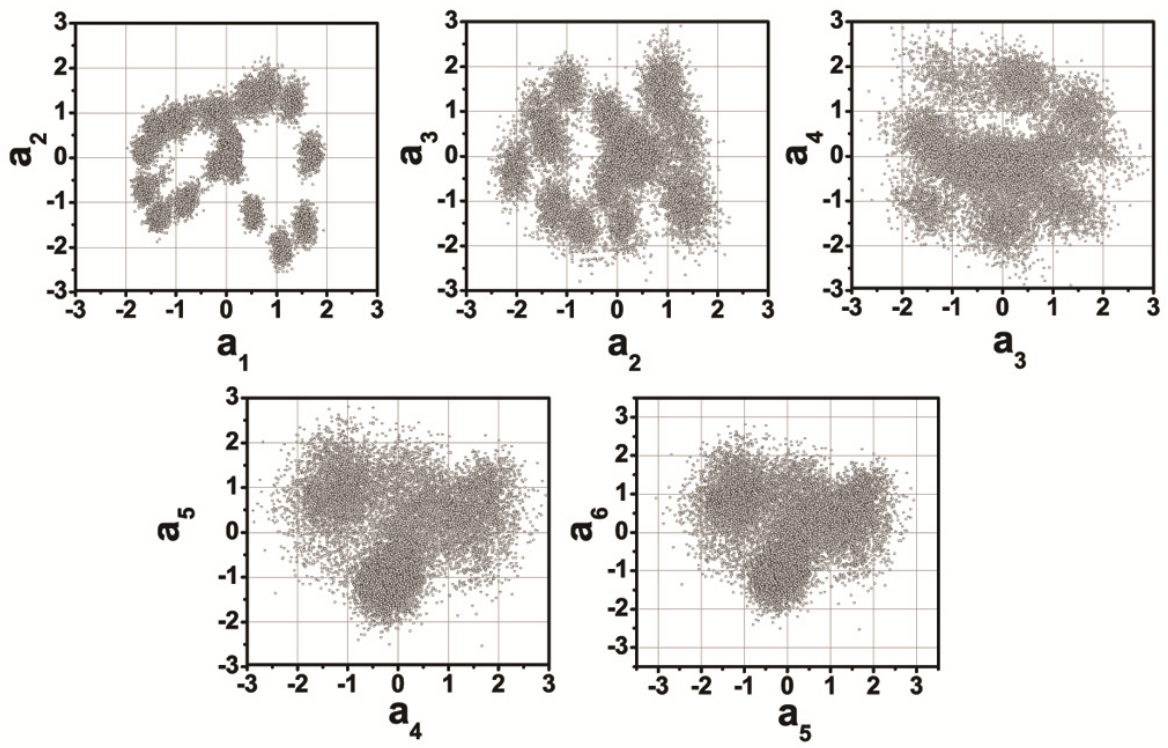

Figure 10. Portrait of phase of the expansion coefficients $a_{1}=f\left(a_{2}\right), a_{2}=f\left(a_{3}\right), a_{3}=f\left(a_{4}\right), a_{4}=f\left(a_{5}\right)$, and $a_{5}=f\left(a_{6}\right)$. 
Some of the spatial modes are depicted in Figure 11. The first mode presents some similarities with the mean volume force, the main common character being the covered region that corresponds to the plasma discharge and a similar distribution of the force vector field. By considering the high periodicity and the alternation of positive and negative in the corresponding expansion coefficient $a_{1}$, this mode is the main contributor to the unsteady and periodic characters of the EHD volume force. According to Figure 11, modes from 2 to 4 relate to the vertical component of the volume force. This confirms the contribution of the vertical volume force in the unsteady momentum transfer. For spatial modes with a higher rank, no clear contribution to the total volume force can be distinguished.
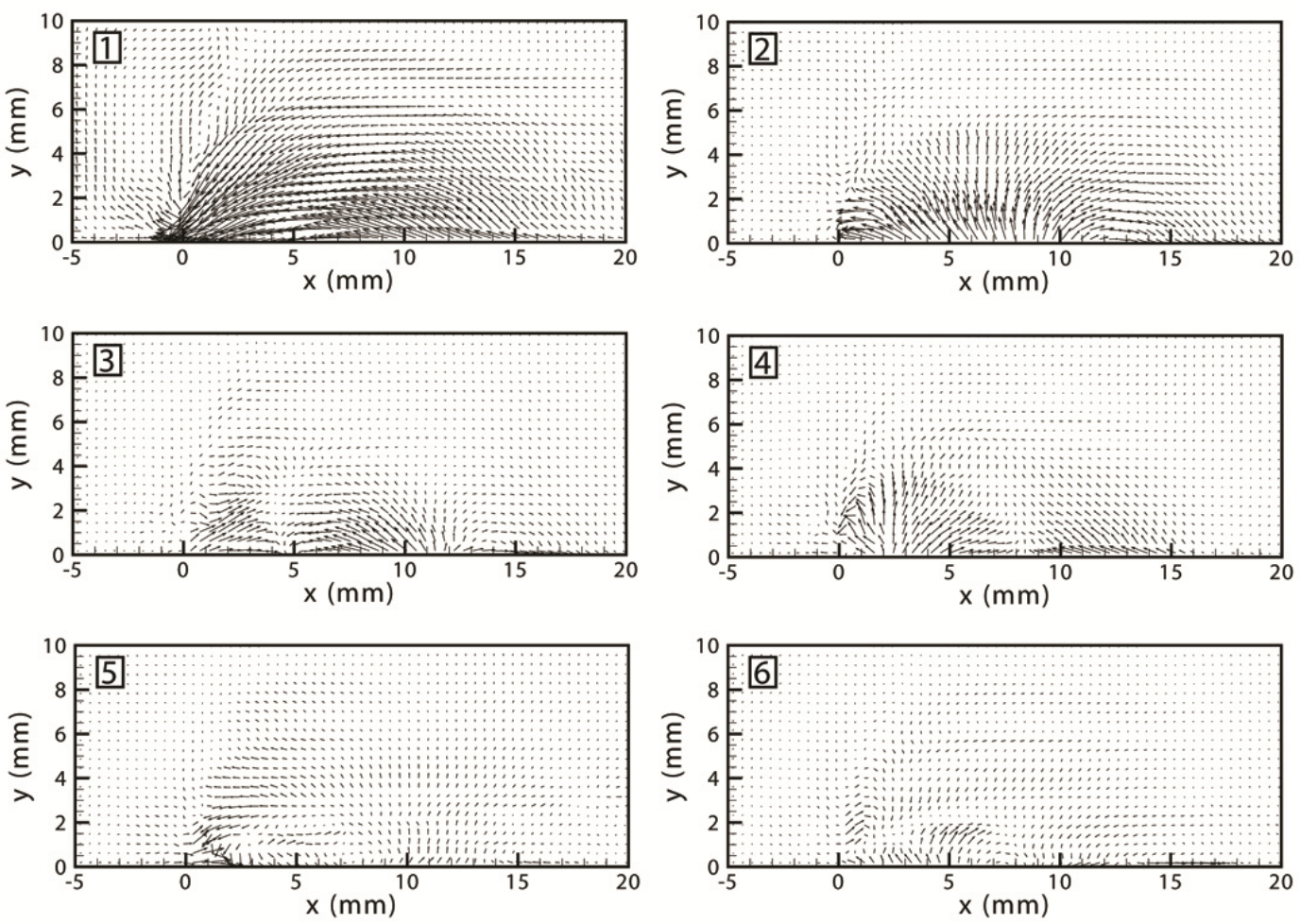

Figure 11. Six first spatial modes computed by proper orthogonal decomposition.

\section{III-2 Selection of the ROM model}

In a first test, equation (5) is used to reconstruct the volume force field with a cut-off level $\mathrm{M}$ fixed at different values (with the mean volume force being superimposed). The reconstruction has been performed with the first mode alone or with the two first POD modes since their frequency contents are correlated. Four modes are also considered in agreement with Figure 11 where the dominant contribution of these four modes has been shown. Finally, the volume force is estimated by considering 16 POD modes this latter situation defining a reference case. The results are shown for one phase of the ac cycle, the one for that the volume force $F_{x}$ is maximized (Figure 12).

The reconstructed force looks almost similar when the first or two first POD modes are considered, the main difference being higher amplitude when two modes are included in the model. The use of 6 POD modes modifies the spatial distribution of $F_{x}$ with a significant component just downstream of the propagation of the glow regime (i.e. $5<x<10 \mathrm{~mm}$ ). By using 4 or 6 POD modes, the topology of 
the resulting volume force is in good agreement with a volume force composed of 16 POD modes but smoother contour are observed by using 4 POD modes. It seems that a truncation at $\mathrm{M}=4$ is an accurate choice regarding the results in Figure 12 but also according to the analysis of the expansion coefficients and spatial modes.

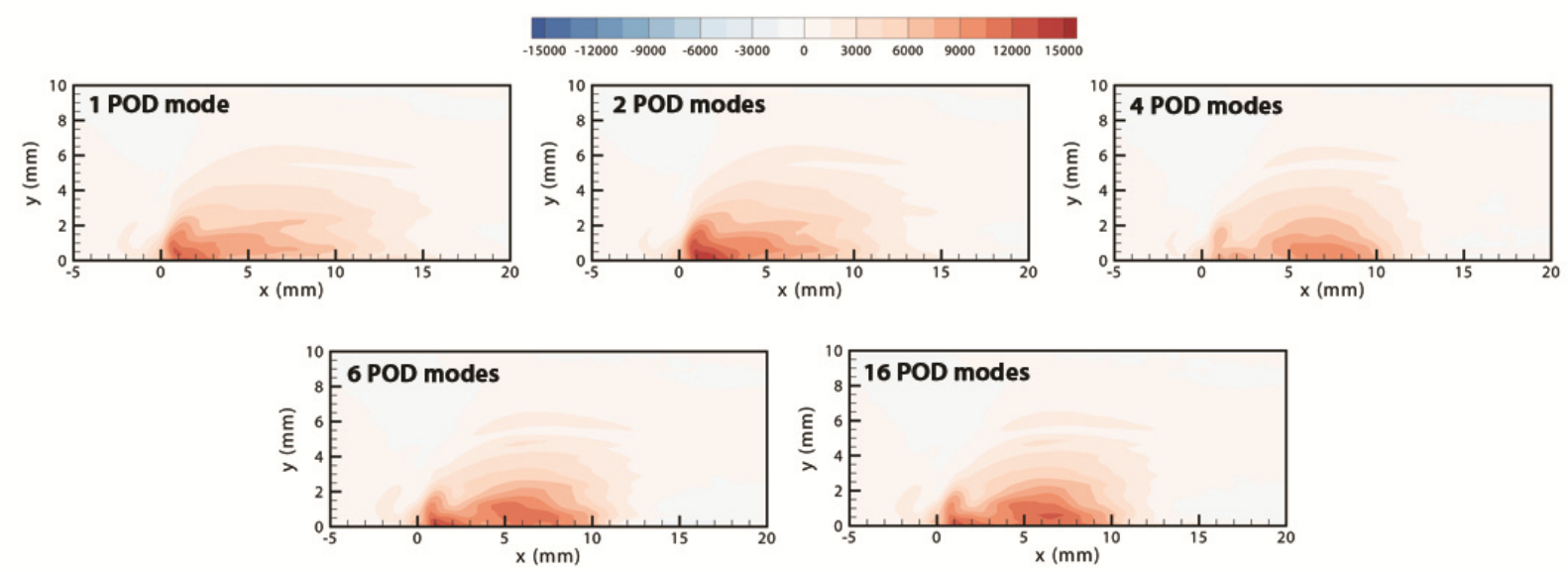

Figure 12. Reconstruction by POD of one instant of the EHD volume force cycle $\left(F_{x}\right)$ with a considered number of modes from 1 to 16 .

\section{III-3 Dynamic reduced order model of the EHD volume force}

The model of the EHD volume force is reconstructed by using the first four POD modes plus the mean volume force distribution. By this method, the data are filtered in space and time but the model includes the dynamic aspects of the plasma discharge. This model can be implemented in a numerical solver by using directly the database issuing from this research or, in a near future, by using a mathematical formulation defined by an effective parametrization of the available data. Nevertheless, the time dependence of the EHD volume force is commented in the last part of this paper,. The results are presented separately depending on their capabilities to produce a positive or negative volume force.

The instants of one ac period at which a positive volume discharge is observed are plotted together in Figure 13. These instants are continuous and they cover the time period from $0.45 \mathrm{~ms}$ to $0.75 \mathrm{~ms}$. This period corresponds exactly to the glow regime of the discharge, a regime universally recognized as the source for the production of momentum transfer (see [2]). At the first instants of the glow regime $(\mathrm{t}<0.5 \mathrm{~ms})$, both components of the estimated volume force are small. When the glow regime is established, a positive force forms for both components. This force covers the first $5 \mathrm{~mm}$ downstream of the edge of the top-electrode. The force is maximal at time equal to $0.55 \mathrm{~ms}$ (phase 3 in Figure 13). From times equal to $0.55 \mathrm{~ms}$ up to $0.75 \mathrm{~ms}$ (phases 4 to 7 ), the force $F_{x}$ propagates in the upstream direction with a significant dissipation illustrated by the enlargement of the region under the influence of the force and the reduced magnitude with time. The secondary component of the volume force also exhibits a progressive increase in amplitude with time. The amplitude of $F_{y}$ is of a same order than $F_{x}$, but it is localized only in the region at the edge of the top electrode. In the first instants of the glow regime (from phase 1 to 3), this component corresponds to an "ejection" of fluid from the top-electrode, this contributing for the superimposition of a wavy perturbation whose fundamental frequency is exactly the one of the applied frequency $\mathrm{f}_{\mathrm{AC}}$. Later, a region of negative force forms concomitantly to the region of positive values, these two regions with opposite sign contributing to a fluid suction/ejection in the close proximity of the top-electrode where the electric field should be maximized. 

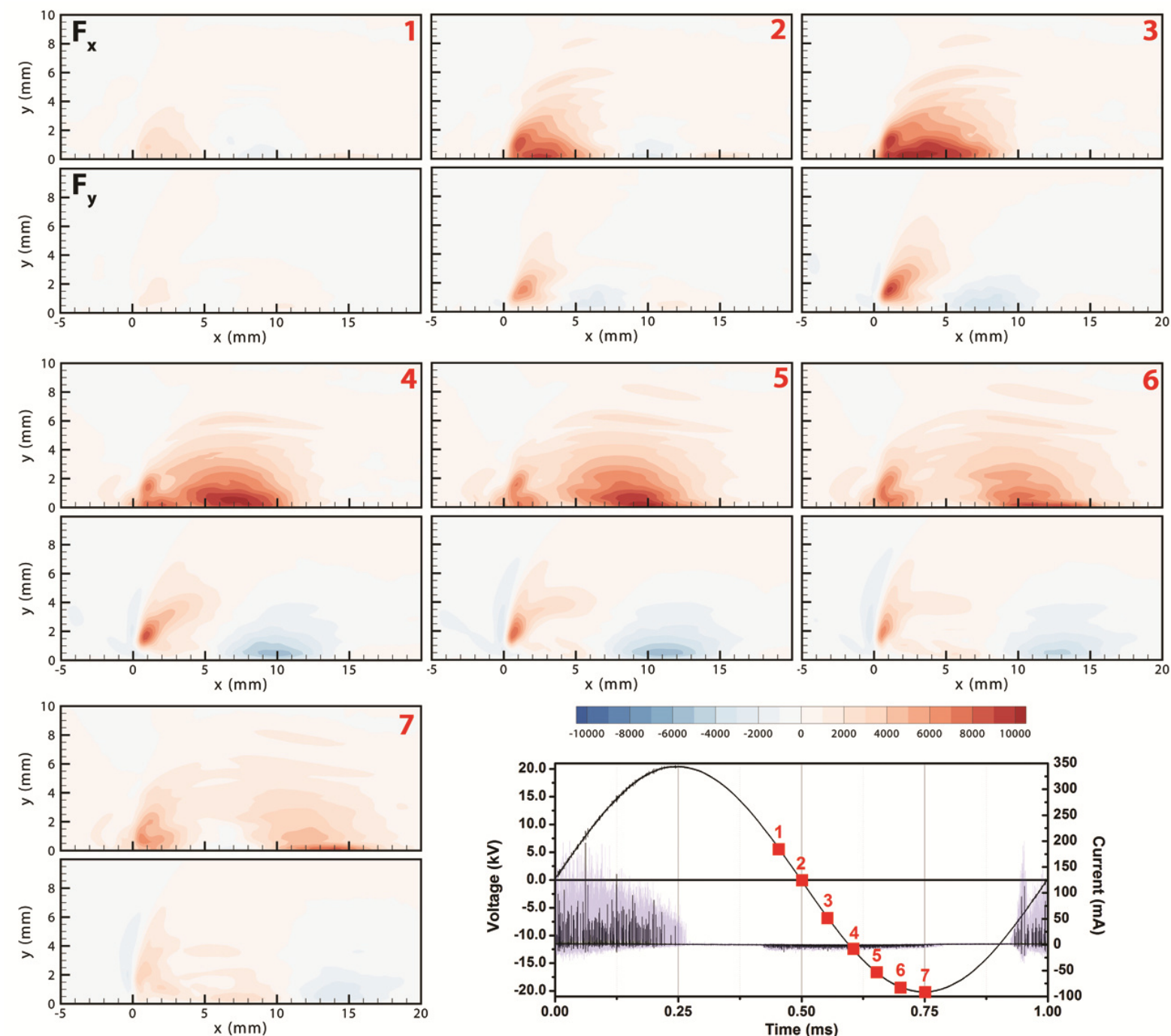

Figure 13. Time evolution of the volume force for the instants responsible for a positive $F_{x}$ (glow regime of the surface DBD).

The times at which a negative volume force dominates the spatial domain are illustrated in Figure 14. The negative volume force forms consecutively to the dissipation of the positive volume force. The dynamic of the negative volume force $F_{x}$ looks almost similar to that of the positive one. The force enlarges and amplifies from the top-electrode and it progressively dissipates after having propagated up to the end of the plasma extension. The $F_{y}$ component is smaller than $F_{x}$ but a negative region is also observed in phase 10 and following phases. This volume force initiates at the same time than the streamer regime and it shows a force directed to the dielectric wall from the top-electrode in the first instants and then the region extends up to $\sim 10 \mathrm{~mm}$ with the time.

For the phases not shown in this paper, the volume force is weak indicating that the EHD force has been largely dissipated at these times. One discussion point is the generation of a volume force at the phases labelled as 8 and 9 in Figure 14, a force that is not expected at this time. Indeed, at these instants the electrical current does not show any trace of electrical activity and no light emission can be observed even with iCCD imaging. In such a condition, the EHD force should be almost null. However, one has to remind that the EHD volume force is only estimated here, the assumption being 
that the pressure gradient is negligible. In fact, the term $\boldsymbol{F}-\nabla p$ is deduced from the time-resolved PIV. While valid in most of the phase instants, the method, and it inherent assumption, could be no more verified for some phases of the EHD volume force. Here, it is suspected that the negative volume force $F_{x}$ is caused by the pressure gradient resulting from the sudden flow deceleration at the end of the glow regime. Because of the good temporal synchronization between the first streamers and the negative volume force $\mathrm{F}_{\mathrm{y}}$ and also because of an expected low $\partial p / \partial y$ for the deceleration of a flow massively convected in the $\mathrm{x}$ direction, it is concluded that the streamer regime could be responsible for the volume force $\mathrm{F}_{\mathrm{y}}$ directed toward the dielectric wall.
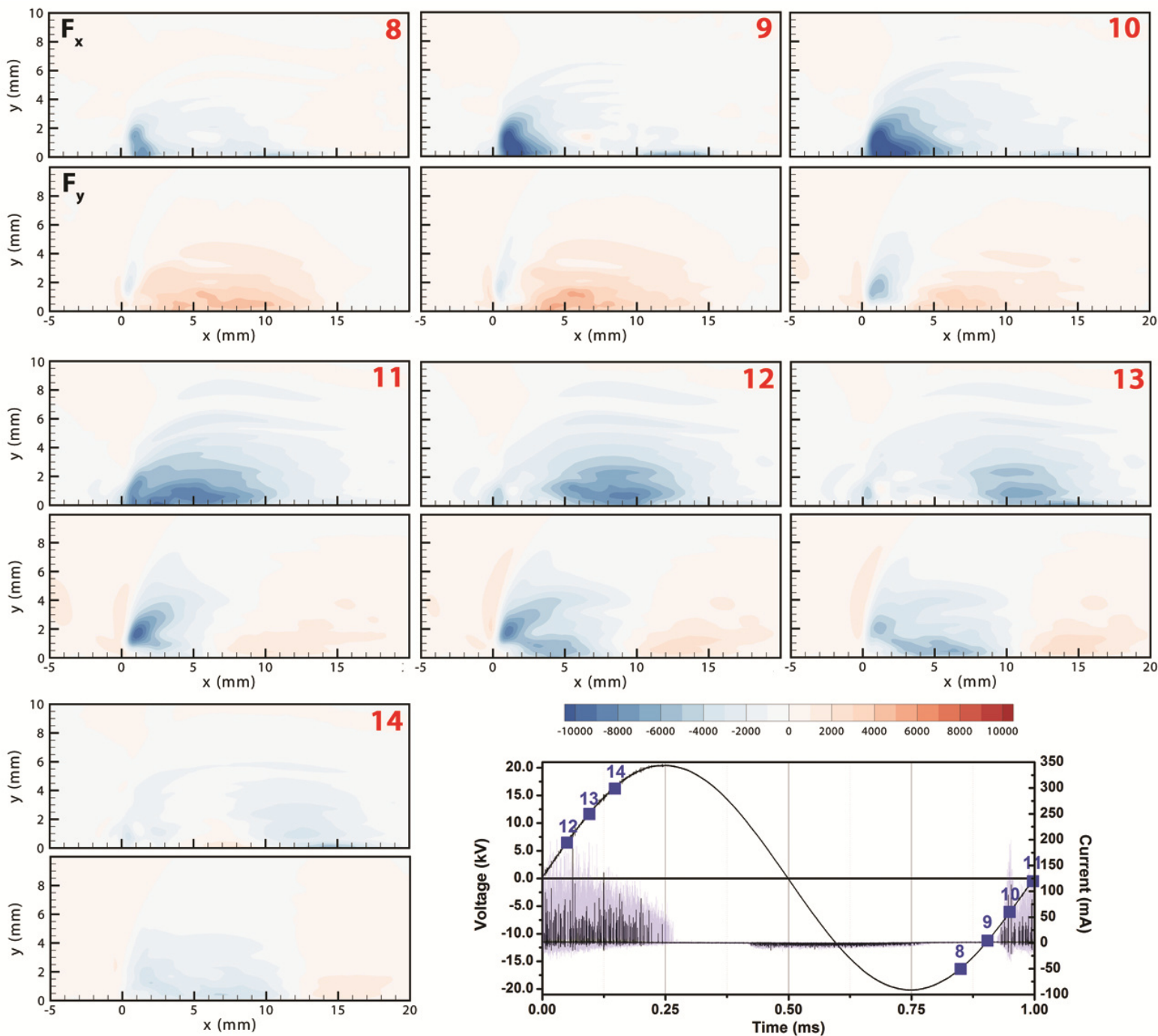

Figure 14. Time evolution of the volume force for the instants responsible for a negative $F_{x}$.

\section{Summary}

The time-dependent behavior of the EHD volume force has been estimated by reversing the Navier-Stokes equations as done in [26-28]. In view of the use of this force as a source term in a numerical CFD solver, a reduced order model has been defined. This investigation has shown that the 
main part of the energy of the EHD force can be described by a few modes only when proper orthogonal decomposition is applied. The first two modes are highly periodic and strongly crosscorrelated and they are responsible for most of the dynamic of the EHD force. However, the present study indicates that a minimum of 6 modes is necessary for a better fidelity to the original data. The resulting reduced order model has been presented. The good temporal resolution gives details on the EHD force production over one period of the applied electrical signal. The positive EHD volume force is produced in the plasma regime where a glow discharge develops from the top-electrode. This is in full agreement with many studies. Here, the positive volume force is immediately followed by a negative force, this in absence of any potential EHD phenomenon. It is concluded that the PIV-based method for the estimation of the volume force may be not valid during the sudden and local flow deceleration when the glow regime extinguishes. In such a flow deceleration, the pressure gradient can be high while the EHD force is, at least low, or null. The results in this study show the limit of the PIV-based approach as it is defined here but a complete discussion and demonstration of the validity of the method is out-of-the-scope of the present paper. This paper is a preliminary stage in defining a reduced order model of a DBD plasma actuator, the coming stage being its implementation into a high-order DNS code for a full validation. However, complementary investigations are under progress to improve the PIV-based approach when the produced flow field is time-resolved and electrical measurements available.

\section{Acknowledgment}

Nicolas Benard and Eric Moreau would like to thank the French ANR program and DGA under reference ANR-13-ASTR-0005 for the SINAPS project. A part of the experimental equipment used in this work was funded by the French Government program Investissements dAvenir (LABEX INTERACTIFS, reference ANR-11-LABX-0017-01). Finally, the authors would like to acknowledge the NATO Applied Vehicle Technology (AVT) 254 "Assessment of Plasma Actuator Technologies for Internal Flows".

\section{References}

1. Corke, T.C., Enloe, C.L., Wilkinson, S.P., 2010. Dielectric barrier discharge plasma actuators for flow control,. Ann. Rev. Fluid Mech, Vol.. 42, pp. 505-529.

2. Benard, N., Moreau, E., 2014. Electrical and mechanical characteristics of surface AC dielectric barrier discharge plasma actuators applied to airflow control. Exp. Fluids, Vol. 55, pp. 1-43.

3. Wang J.J., Choi K.S;, Feng L.H., Jukes T.N. 2013. Recent developments in DBD plasma flow control., Prog Aerosp Sci., Vol. 62, pp. 52-78

4. Kriegseis J., Simon B., and Grundmann S., Towards In-Flight Applications? A Review on Dielectric Barrier Discharge-Based Boundary-Layer Control., Appl. Mech. Rev, Vol. 68, 020802, 2016

5. Kogelschatz U., 2003. Dielectric-barrier Discharges: Their History, Discharge Physics, and Industrial Applications., Plasma Chemistry and Plasma Processing, Vol. 23, No. 1.

6. Boeuf J., Lagmich Y., Unfer T., Callegari T., and Pitchford L. 2007. Electrohydrodynamic force in dielectric barrier discharge plasma actuators., J. of Phys. D: Applied Physics, Vol. 40, pp. 652.

7. Jayaraman B., and Shyy W. 2008. Modeling of dielectric barrier discha rge-induced fluid dynamics and heat transfer., Prog. in Aerosp. Sci., Vol. 44, pp. 139 - 191

8. F. Rogier, G. Dufour, and K. Kourtzanidis. 2014. Numerical simulation of sinusoidal driven dbd actuators and comparisons with experiments. In AIAA pap er 2014-2808

9. H Nishida, T. Nonomura, and T. Abe. 2016. Numerical study on spanwise nonuniformity in bodyforce field of dielectric-barrier-discharge plasma actuator. AIAA Journal, Vol. 54, pp.659-669.

10. K. P. Singh and S. Roy. 2008. Force approximation for a plasma actuator operating in atmospheric air., J. of Applied Phys., 103(1):013305, 2008.

11. Y.B. Suzen, P.G. Huang, and D.E. Ashpis. 2007. Numerical simulations of flow separation control in low-pressure turbines using plasma actuators., In AIAA paper 2007-937, 2007. 
12. Jayaraman B., Cho Y.C., and Shyy W., 2008. Modeling of dielectric barrier discharge plasma actuator., J. Appl. Phys. 103, 053304

13. Enloe CL, McLaughlin T, Van Dyken R, Kachner K, Jumper E, Corke TC, 2004. Mechanisms and responses of a single dielectric barrier plasma actuator: plasma morphology., AIAA J., Vol. 42, pp. 589-594

14. Mertz B.E. and Corke T.C. 2011. Single-dielectric barrier discharge plasma actuator modelling and validation. J. Fluid Mech., Vol. 669, pp. 557-583

15. D.M. Orlov, T.C. Corke, and M. Patel., 2006. Electric circuit model for aerodynamic plasma actuator. In AIAA paper 2006-1206

16. Kotsonis M., Ghaemi S., Giepman R., and Veldhuis L. 2010. Experimental study on the body force field of dielectric barrier discharge actuators., AIAA paper 2010-4630

17. Debien A, Benard N, David L, Moreau E. 2012. Unsteady aspect of the electrohydrodynamic force produced by surface dielectric barrier discharge actuators. Appl Phys Lett 100:013901

18. Kotsonis M. 2015. Diagnostics for characterisation of plasma actuators. Measurement Science and Technology, Vol. 26, 092001

19. Pescini E., Martínez D., De Giorgi M.G. and Ficarella A., 2015. Optimization of micro single dielectric barrier discharge plasma actuator models based on experimental velocity and body force fields, Acta Astronautica

20. Maden I, Maduta R., Hofmann J., Jakirlić S., Kriegseis J., Tropea C., and Grundmann S. 2016. Modelling the Plasma-actuator-related Turbulence Production in RANS Closures by Reference to Complementary Experimental Investigations. Flow, Turbulence and Combustion, Vol. 97, pp. 10471069

21. Wilke B., 2009. Aerodynamische Strömungssteuerung mittels dielektrischen BarriereentladungsPlasmaaktuatoren. PhD Thesis, DLR Göttingen, Germany

22. Kotsonis M., Ghaemi S., Veldhuis L., and Scarano F. 2011. Measurement of the body force field of plasma actuators. J. of Phys. D: Applied Physics, Vol. 44, pp. 045204.

23. Kriegseis, J., Schwarz, C., Tropea, C., Grundmann, S., 2013. Velocity-information based force-term estimation of dielectric barrier discharge plasma actuators. Journal of Physics D: Applied Physics, Vol. 46, pp. 055202

24. Maden I., Maduta R., Kriegseis J., Jakirli’c S., Schwarz C., Grundmann S., and Tropea C. 2013. Experimental and computational study of the flow induced by a plasma actuator. Int. J. Heat and Fluid Flow, 41:80-89.

25. Benard N., Caron M., and Moreau E. 2015. Evaluation of the time-resolved ehd force produced by a plasma actuator by particle image velocimetry- a parametric study. In J. of Phys.: Conference Series, Vol. 646, pp. 12055-12058.

26. Benard N, Debien A, Moreau E. 2013. Time-dependent volume force produced by a non-thermal plasma actuator from experimental velocity field. J Phys D Appl Phys, Vol. 46, pp. 245201

27. Neumann M., Friedrich C., Czarske J., Kriegseis J., and Grundmann S. 2013. Determination of the phase-resolved body force produced by a dielectric barrier discharge plasma actuator., J. of Phys. D: Appl. Phys., Vol. 46, pp. 42001

28. Kuhnhenn, M., Simon, B., Maden, I. Kriegseis, J. 2016. Interrelation of phase-averaged volume force and capacitance of DBD plasma actuators., J. Fluid Mech.,Vol. 809, pp. 1-11

29. Brauner T., Laizet S., Benard N., and Moreau E. 2016. Modelling of Dielectric Barrier Discharge Plasma Actuators for Direct Numerical Simulations., AIAA paper 2016-3774

30. Benard N., Noté P., Caron M., and Moreau E. 2017. Influence on the electric wind velocity of the frequency of a sinusoidal high voltage powering a surface DBD plasma discharge., To be published in Journal of Electrostatics

31. Sirovich L. 1987. Turbulence and the dynamics of coherent structures., Part 1,2,3. Quart Appl Math, Vol. 3, pp. 561-571

32. Cordier L., Bergmann M. 2003. Proper orthogonal decomposition: an overview., Lecture series 2003-03 in VKI. Post-processing of experimental and numerical data, vol 2 